\title{
COMPARACIÓN DE LA CALIDAD DEL HENO DE PASTO TRANSVALA (Digitaria decumbens cv. Transvala) PRODUCIDO BAJO RIEGO, HENO DE PASTO TRANSVALA COMERCIAL Y PACAS DE PAJA DE ARROZ, MEDIANTE LA GANANCIA DE PESO DE TORETES ESTABULADOS
}

\author{
Jorge L. Morales González¹, Carlos Hidalgo Ardón ${ }^{1}$, Argerie Cruz Méndez. ${ }^{1}$
}

\begin{abstract}
RESUMEN
La calidad de un forraje se determina, principalmente, por medio de la respuesta productiva de los animales (Ball et al. 2001). El presente estudio tuvo como objetivo, comparar la calidad del heno de pasto Transvala (Digitaria decumbens cultivar Transvala) producido bajo condiciones de riego, fertilizado y cosechado a 45 días de rebrote, con la de pacas comerciales tanto de Transvala como de paja de arroz, mediante la ganancia de peso y el consumo de estos forrajes por toretes estabulados. Veintisiete toretes con un peso promedio de $282 \mathrm{~kg}$ de peso vivo (PV) fueron estabulados por 77 días y alimentados con heno de pasto Transvala de 45 días de rebrote (HBC), heno comercial de pasto Transvala (HCM) y paja de arroz (PAR). Los dos últimos tratamientos con acceso además a $0,5 \mathrm{~kg}$ de melaza y $30 \mathrm{~g}$ diarios de urea. El forraje se dio a libre consumo. Los datos se analizaron mediante un diseño experimental irrestricto al azar. Los toretes alimentados con HBC lograron pesos finales mayores $(304,8 ; 295,5$ y $288,5 \mathrm{~kg} ; \mathrm{P}<0,01)$ que los otros dos grupos de animales; así como consumos de forraje, con base en materia seca (kg MS/100 kg PV) superiores $(2,26,2,08$ y 1,79 , respectivamente; $\mathrm{P}<0,01)$ a los logrados con HCM y PAR. Los contenidos (\%) de: proteína cruda (PC), fibra neutro detergente (FND) y fibra ácido detergente (FAD) de HBC, HCM y PAR fueron $9,0,64,4$ y 43,3; 4,1, 63,4 y 46,2; 4,1, 60,5 y 53,6, respectivamente. La respuesta animal observada confirma los parámetros de calidad obtenidos en el laboratorio, particularmente en relación con el contenido de PC y permite concluir que el heno de pasto Transvala producido bajo condiciones de riego y fertilizado, a una edad de rebrote de 45 días es superior en calidad a los henos comerciales de pasto Transvala y a la paca de arroz, disponibles en el mercado.
\end{abstract}

Palabras clave: heno de calidad, pasto Transvala, riego, ganancia de peso.

\section{INTRODUCCIÓN}

El mercado tradicional de heno en Costa Rica, está sustentado principalmente por la demanda en épocas críticas, debido a la baja disponibilidad de forrajes, ocasionada por la estacionalidad climática. La época de verano en el Pacífico Seco es el periodo de mayor demanda, el cual se ve acentuado cíclicamente por el fenómeno del Niño que prolonga el periodo seco y/o intensifica las lluvias en las zonas de trópico húmedo. Por esto se dice que el mercado del heno es un mercado de crisis para mantener animales y no un mercado regulado por la oferta y la demanda, para la producción animal (Morales 2000).

Estas características del mercado del heno en Costa Rica no han permitido establecer estándares de calidad en el producto. Evidencia de esto, es que la única referencia de calidad y

\footnotetext{
${ }^{1}$ Instituto Nacional de Innovación y Transferencia en Tecnología Agropecuaria (INTA), Costa Rica.
} 
que determina un precio diferenciado es, si el origen de dicho forraje es de paja de arroz ó de pasto Transvala y dentro de éste último, la aceptación o rechazo a un precio determinado, está dado por el nivel de contaminantes que contenga, llámense malezas u otro tipo de materiales diferentes al pasto Transvala (Morales et al. 2003). Aquí interviene también el caballista, un comprador dispuesto a pagar precios altos, particularmente de pacas de pasto Transvala, pero bajo los mismos criterios de calidad mencionados anteriormente.

Trabajos de investigación realizados por el INTA en mejoramiento de la calidad del heno (Morales et al. 2006 a y b), han resultado en tecnologías que permiten triplicar el contenido nutricional del forraje y su productividad. Un forraje conservado en forma de heno, con contenidos de proteína cruda y digestibilidad (DIVMS) cercanos al $10 \%$ y $60 \%$, respectivamente, permite la producción animal y no sólo el mantenimiento de peso, como ocurre con las pacas comerciales que actualmente se encuentran en el mercado.

Se ha comprobado que muchas veces el valor nutritivo de la paja de arroz es superior al de la paca de pasto Transvala comercial (Morales et al. 2003). Productores y comerciantes de estas pajas saben que estas sirven únicamente para que los animales pierdan menos peso durante la época seca y que se debe suministrar además, melaza y urea, de lo contrario no servirán ni para ese propósito. Por lo tanto, ni pensar que estas pajas sirvan para producir; tal vez como fuente de fibra en sistemas estabulados, particularmente aquellos de producción de leche.

Tal parece que el poco desarrollo de la henificación en el país se debe, entre otros factores, a problemas de calidad forrajera y por ello exista una demanda real por estos forrajes, exclusivamente durante periodos críticos.

Es factible aumentar tanto la producción como la calidad del heno en sistemas bajo riego, en comparación con el sistema tradicional de secano (Morales et al. 2006 a, b). Para cambiar la cultura tradicional en la producción y uso de los henos y abrir un mercado más amplio basado en la calidad, se debe demostrar, el efecto que sobre la producción animal tiene el suministro de heno de buena calidad a los animales. Por lo anterior, el presente estudio tuvo como objetivo determinar el potencial real para la producción animal del heno de pasto Transvala, producido bajo condiciones de riego, fertilizado y cosechado a 45 días de rebrote, en comparación con henos tradicionales.

\section{MATERIALES Y MÉTODOS}

\section{Ubicación del Ensayo}

El presente estudio se llevó a cabo en los corrales de engorde de la Estación Experimental Enrique Jiménez Nuñez (E.E.E.J.N.), del INTA, ubicada en el cantón de Cañas, Guanacaste, $10 \mathrm{~km}$ al sureste del distrito central y $10 \mathrm{~km}$ al sur de la carretera Panamericana. Sus limitantes están relacionadas con la Zona de Vida de Bosque Húmedo, transición a Basal y Tropical, dadas por fuertes vientos y un patrón climático bimodal, con una estación seca de diciembre a abril y un invierno intenso en lluvias, de setiembre a mediados de noviembre, separados por un periodo que se ha manifestado errático en los últimos años, ligero de lluvias de mayo a mediados de julio y de aquí a agosto con un lapso de un mes en el que se reduce la precipitación significativamente, correspondiente al veranillo de San Juan y a la canícula.

La Figura 1 muestra el comportamiento del clima en la zona (Hancock y Hargreaves 1977), el cual está basado en datos de seis años y en

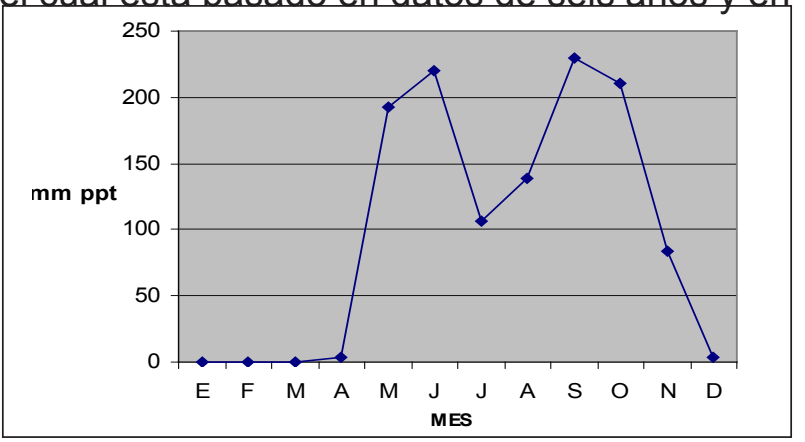

probabilidades de un $75 \%$ de que este patrón de lluvias pueda ocurrir.

Figura 1. Patrón de lluvias con $75 \%$ de probabilida- 
des para el área de la E.E. E.J.N. Guanacaste, Costa Rica. 2000.

La temperatura promedio de la zona ha sido de $24,6{ }^{\circ} \mathrm{C}$; la humedad relativa de $91 \%$ y la precipitación máxima y mínima fue de $2.818 \mathrm{~mm}$ y $1.018 \mathrm{~mm}$, respectivamente, durante el mismo lapso. El ensayo se realizó del 3 de marzo al 17 de junio del 2000.

\section{Tipos de Henos}

El heno de 45 días de rebrote de pasto Transvala (HBC), utilizado en este estudio, fue producido, en un sistema de producción bajo riego y fertilizado con fórmula completa 10-30-10 a una dosis de $50 \mathrm{~kg}$ de $\mathrm{P}_{2} \mathrm{O}_{5}$, en una sola aplicación para todo el periodo y nutrán, a una tasa de fertilización de 103,5 kg de N/ha/corte. Para ello, los campos de pasto Transvala fueron establecidos en junio de 1999 y uniformados entre el 13 y 15 de octubre para obtener heno de 45 días de rebrote.

El heno comercial de pasto Transvala (HCM) se adquirió de productores que producen bajo condiciones de secano en el área de Cañas. La paja de arroz (PAR) se consiguió de la paca comercial disponible en la misma área.

\section{Animales utilizados}

Se utilizaron 27 toretes de alto encaste Brahman con un peso promedio de $282 \mathrm{~kg}$ de peso vivo (Cuadro 1), provenientes de la Estación Experimental Los Diamantes, del INTA localizada en Guápiles, región Atlántica de Costa Rica. Se colocaron tres animales por corral, correspondiendo a un tratamiento, con tres repeticiones (corrales) por tratamiento, es decir se utilizaron nueve animales por tratamiento. Los animales se sometieron durante 14 días a una fase de adaptación a las dietas. Inmediatamente después entraron a la fase experimental por 61 días. Los animales se pesaron a rumen lleno al inicio de la fase experimental y posteriormente a los 30 y a los 61 días, por las mañanas antes del primer suministro de alimento.

\section{Dietas ( tratamientos)}

Las dietas utilizadas fueron:

T1. Heno de pasto Transvala (HBC) de 45 días de rebrote como única fuente de alimento.

T2. Heno de pasto Transvala comercial (HCM) con $0,5 \mathrm{~kg}$ de melaza y 30 gramos de urea por animal por día.

T3. Paja de arroz (PAR) con 0,5 kg de melaza y 30 gramos de urea por animal por día.

El suministro de urea y melaza ofrece la posibilidad de mejorar la utilización de los alimentos fibrosos, en particular de aquellos de baja calidad como el HCM y PAR, mediante la disponibi-

Cuadro 1. Peso inicial y promedio en kilogramos de los animales por tratamiento al inicio de la fase experimental de evaluación. Guanacaste, Costa Rica. 2000.

\begin{tabular}{|c|c|c|c|c|c|c|c|c|c|}
\hline \multirow{3}{*}{$\begin{array}{c}\text { Repeticiones } \\
1\end{array}$} & \multicolumn{9}{|c|}{ Tratamientos } \\
\hline & \multicolumn{3}{|c|}{ HBC } & \multicolumn{3}{|c|}{ HCM } & \multicolumn{3}{|c|}{ PAR } \\
\hline & 313 & 320 & 311 & 353 & 325 & 289 & 312 & 297 & 277 \\
\hline 2 & 289 & 255 & 297 & 287 & 273 & 323 & 269 & 266 & 279 \\
\hline 3 & 255 & 270 & 295 & 200 & 252 & 205 & 242 & 278 & 282 \\
\hline Promedio & & 289,5 & & & 278,5 & & & 278,0 & \\
\hline Promedio total & & & & & 282 & & & & \\
\hline
\end{tabular}


lidad de nutrientes deficitarios en dichos forrajes para el crecimiento de la población microbiana del rumen (Leng et al. 1977, Leng 1990).

La fuente de forraje fue suministrada diariamente a libre consumo en los tres tratamientos. La urea se disolvió en 20 cc de agua tibia y se mezcló con la melaza diariamente. Los animales fueron desparasitados internamente con 15 cc de Levamisol, vitaminados con $4 \mathrm{cc}$ de complejo ADE y 7 cc de Butafosfan, un compuesto de vitamina B12 y fósforo. Igualmente todos tuvieron libre acceso a una mezcla mineral y agua.

\section{Diseño Experimental}

EL estudio se arregló como un diseño irrestricto al azar, con tres repeticiones (corrales) y tres animales por repetición. El análisis de los datos se hizo mediante el paquete estadístico SAS (SAS 1984).

\section{Variables dependientes}

Las variables dependientes medidas fueron peso final de los animales y consumo diario de las dietas. Los pesos finales se ajustaron por covarianza utilizando los pesos iniciales de los animales.

\section{Variables independientes}

Las variables independientes fueron los tipos de forrajes, los cuales se caracterizaron mediante sus contenidos de proteína cruda y fibra.

\section{Muestreos}

En todos los lotes de forraje de heno de Tansvala de 45 días de rebrote y henos de Transvala comercial y paja de arroz, se tomaron muestras de $500 \mathrm{~g} \mathrm{c} / \mathrm{u}$. Posteriormente, se sometieron a secado en horno a $60{ }^{\circ} \mathrm{C}$ por 72 horas y se enviaron al laboratorio para el análisis respectivo.

\section{Análisis de Laboratorio}

En el laboratorio de Piensos y Forrajes del INTA, las muestras se secaron en horno a 105
${ }^{\circ} \mathrm{C}$, durante 24 horas, para determinar materia seca y después se molieron en molino de martillos utilizando una malla de $2 \mathrm{~mm}$. Se analizaron los contenidos de: proteína cruda (\% PC), por el método de micro Kjeldahl, y fibra ácido detergente (\% FAD) y fibra neutro detergente (\% FND), utilizando la metodología de Van Soest (1967).

\section{RESULTADOS Y DISCUSIÓN}

\section{Contenido nutricional del forraje}

Las fuentes de forraje utilizadas en este estudio fueron de calidades claramente diferentes, como se puede observar en el Cuadro 2. Estas diferencias son originadas básicamente por el estado de madurez de la planta al ser cosechada para heno (Arthington y Brown 2005). El heno HBC fue cosechado a una edad aproximada de 45 días de rebrote, el heno $\mathrm{HCM}$ a más de 90 días y la paca PAR es simplemente el residuo de la cosecha de arroz, el cual se cosecha a más de 120 días después de la siembra.

A mayor madurez de la planta forrajera la composición de la fibra (FND, FAD y lignina) varía. En estados avanzados de madurez, hay una deposición mayor de lignina (Van Soest 1983), que aunque en el presente estudio no se midió, es posible que esté influyendo en la calidad de HCM y PAR, con respecto a HBC. Se sabe que la lignina es un elemento clave en la baja digestibilidad de las paredes celulares de los forrajes y tanto la concentración como la misma digestibilidad de las paredes celulares, afectan negativamente el potencial de consumo y disponibilidad de energía de los cultivos forrajeros (Jung y Allen 1995).

Los tres tipos de forraje utilizados en el presente estudio poseen un contenido de materia seca muy parecida, de alrededor del $85 \%$. Este contenido de humedad en forrajes henificados es similar al que reporta la literatura ( $\mathrm{Li}-$ ppke 1980), y es el que presenta la mayoría de las gramíneas forrajeras, secadas al sol.

En el Cuadro 2, se presenta la calidad nutricio- 
nal de los tres henos utilizados. El contenido de PC, muestra las diferencias de calidad de los tres forrajes. La paca de heno HBC obtenida bajo condiciones de riego a 45 días de rebrote, contiene más del doble de este nutriente que los otros dos forrajes $(4,1 \%)$, resultado observado repetidamente en otros estudios como efecto de la edad de rebrote (Lippke 1980, Arthington y Brown 2005).

Cuadro 2. Calidad de los forrajes evaluados. Guanacaste, Costa Rica. 2000.

\section{TIPO DE FORRAJE}

\begin{tabular}{cccc} 
Variables * $^{*}$ & HBC & HCM & PAR \\
\hline $\mathrm{n}$ & 10,00 & 4,00 & 6,00 \\
$\% \mathrm{MS}$ & 84,42 & 85,40 & 86,12 \\
$\% \mathrm{PC}$ & 9,00 & 4,10 & 4,10 \\
$\% \mathrm{FND}$ & 64,40 & 63,40 & 60,50 \\
$\% \mathrm{FAD}$ & 43,30 & 46,20 & 53,60 \\
$\% \mathrm{HEM}$ & 21,10 & 17,20 & 6,90 \\
\hline \hline
\end{tabular}

${ }^{*} \mathrm{n}=$ número de muestras analizadas; \% MS= contenido de materia seca; \% PC= contenido de proteína cruda; \% FND= contenido de fibra neutro detergente; \% FAD= contenido de fibra ácido detergente; $\% \mathrm{HEM}=$ Hemicelulosa= FND-FAD.

Además, es importante indicar, que el contenido de proteína cruda del heno HCM es igual al que presenta PAR, no por ser una paca de heno de pasto Transvala, se garantiza que sea de buena calidad; hay otros factores más importantes, como la edad de rebrote que intervienen sobre la calidad. También se observa que aún bajo condiciones diferentes y con especies diferentes, a edades de rebrote semejantes, el contenido de proteína cruda es muy similar entre las gramíneas forrajeras, tal como lo reporta Lippke (1980) en pasto Bermuda en Texas, con $8 \%$ de PC a seis semanas de rebrote.

Aunque se presentan sólo ligeras diferencias en el contenido de FND, en HCM y particularmente en PAR, se nota una tendencia a un mayor contenido de FAD que probablemente se sume a sus bajos contenidos de proteína y por lo tanto, incida aún más en sus bajas calidades como fuente de nutrientes para los rumiantes, ya que la FAD es considerada el componente de menor digestibilidad (Cherney y Hall 2008). Ésta es progresivamente menor en el orden PAR > HCM > HBC $(53,60 ; 46,20$ y $43,30 \%$ respectivamente).

La hemicelulosa, en el mismo orden, muestra una tendencia contraria $(6,90 ; 17,20$ y $21,10 \%$ ), es decir, al estar asociada a un mayor consumo y ganancia de peso, pareciera estar relacionada con una mayor digestibilidad.

\section{Consumo de forraje}

La producción de los rumiantes en respuesta a los forrajes, está directamente relacionada con el consumo que puedan hacer de ellos; a mayor consumo, mayor producción. El consumo de forrajes está muy ligado a la capacidad física del rumiante para procesar los materiales fibrosos que como estos, se caracterizan por tener una alta proporción de fibra y que dependiendo de su digestibilidad, podrán ser consumidos en menor o mayor grado. En la fracción de FND se encuentran los componentes indigestibles y lentamente digestibles de las paredes celulares de las plantas (celulosa, hemicelulosa, lignina y cenizas) y en la fracción de FAD se encuentran los mismos componentes de la FND a excepción de la hemicelulosa.

Los niveles de consumo que alcanzaron los animales en los diferentes tratamientos de este estudio, se muestran en el Cuadro 3.

Cuadro 3. Respuesta animal en consumo de materia seca, a la calidad del forraje. Guanacaste, Costa Rica. 2000.

\begin{tabular}{cccc}
\hline & \multicolumn{3}{c}{ Tratamientos** } \\
Variables * & HBC & HCM & PAR \\
\hline Consumo kg /an & $6,89 a$ & $5,99 b$ & $4,92 \mathrm{c}$ \\
Consumo \% PV & 2,30 & 2,10 & 1,75 \\
\hline
\end{tabular}

* an = animal; \% PV = consumo de forraje en base seca como \% del peso vivo. 
** Letras distintas indican que hay diferencia significativa $(p \leq 0,05)$.

La respuesta animal en términos de consumo demuestra las diferencias de calidad entre los diferentes tipos de forrajes utilizados. En valores absolutos $(6,89 \mathrm{~kg}$ de materia seca por día), como en valores relativos $(2,30 \%$ del peso vivo del animal), el consumo de heno HBC superó, a los otros dos forrajes (Cuadro 3).

Es decir, el consumo de materia seca de los diferentes forrajes por los animales, va de mayor a menor según el siguiente orden $\mathrm{HBC}>\mathrm{HCM}>\mathrm{PAR}$. El mayor consumo de HBC probablemente se deba a una mayor digestibilidad en este forraje, ya que es el forraje con menor madurez, mayor contenido de PC y menor contenido de FND por tanto, probablemente posee, una menor deposición de lignina, el componente más indigestible de la fibra que se acumula con la edad de rebrote.

Comparativamente (Figura 2), los animales con $\mathrm{HBC}$ consumieron hasta un $13 \%$ y un $30 \%$ más de materia seca que aquellos con HCM y PAR, respectivamente.

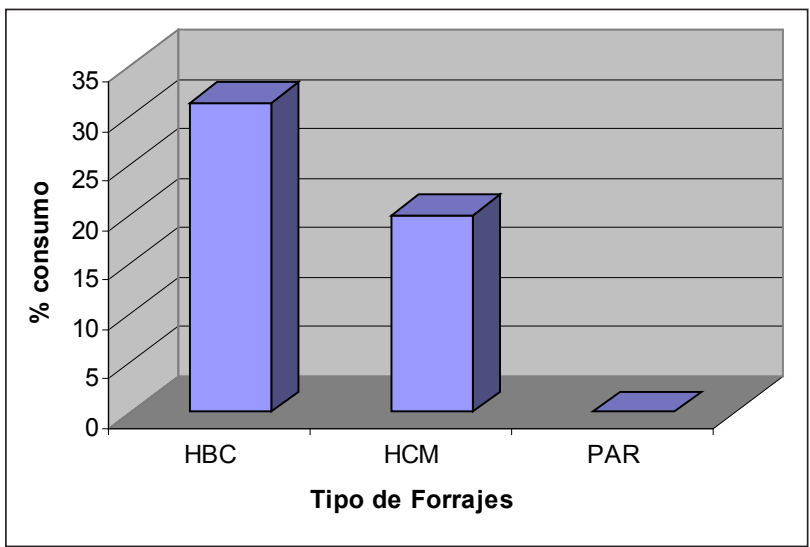

Figura 2. Consumo comparativo de los henos con respecto a la paja de arroz. Guanacaste, Costa Rica. 2000.

Traducido a consumo total de proteína cruda diaria y considerando los ingredientes extra (urea y melaza) suministrados a los animales en los tratamientos HCM y PAR, estos consumieron sólo un 58 \% (360 g) y un $51 \%$ (316 g), respectivamente, de lo consumido por los ani- males en HBC (620 g). Lippke (1980) observó una tendencia muy similar con una correlación significativa $(P<0,01)$ entre contenido de proteína de forraje en forma de heno y consumo de materia seca $(r=0,86)$ en toretes.

Lippke (1980) además reportó, con henos de pasto Bermuda y sorgo, una relación significativa $(r=0,81)$ en el contenido de hemicelulosa y el consumo de FND en toretes; es decir a menor contenido de hemicelulosa, menor el consumo de fibra por el animal. Igualmente con el componente recíproco FAD, Van Soest et al. (1978), reportan una alta relación inversa con el consumo de materia seca por el animal $(r=-0,93$ y $r=-0,61$, respectivamente). Es decir, a mayor contenido de FAD, menor consumo de materia seca. Esos estudios concuerdan con lo encontrado en el presente ensayo ( Figuras 4 y 5$)$.

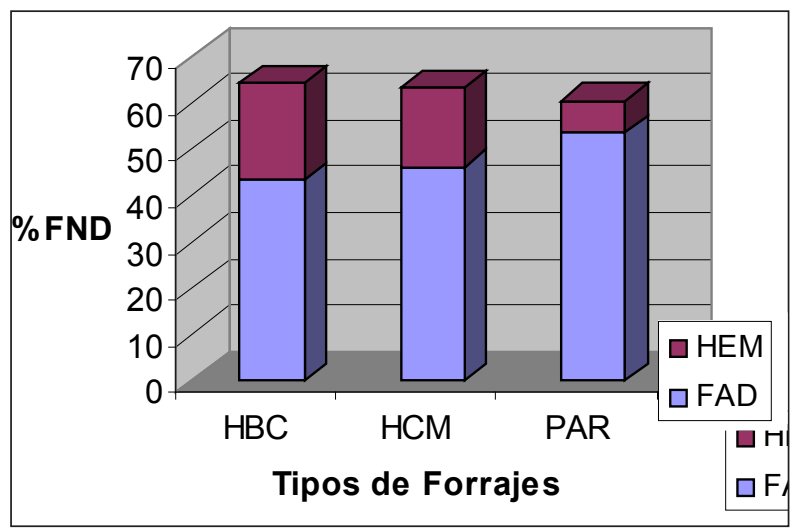

Figura 3. Composición y tendencias de los componentes de la fibra neutro detergente de los diferentes tipos de forrajes. Guanacaste, Costa Rica. 2000.

Estas observaciones de Lippke y Van Soest se encontraron también en el presente estudio, si se correlacionan los datos de la Figura 2 de consumo, con los datos de la Figura 3 que muestra el contenido de las diferentes fracciones fibrosas de los forrajes utilizados. Estas correlaciones se exponen en la Figura 4, en donde en el orden HBC:HCM:PAR, el consumo de MS disminuye conforme el contenido de HEM baja y el contenido de FAD aumenta. 


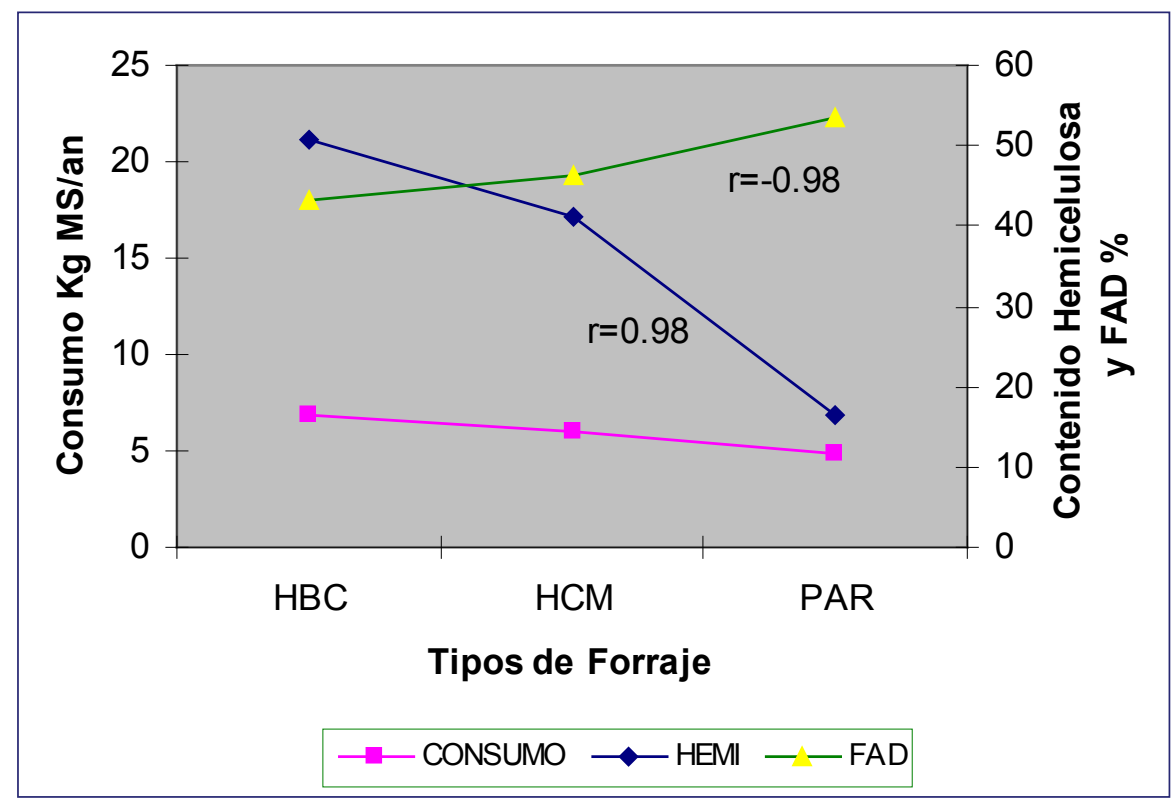

Figura 4. Correlación del consumo, contenidos de hemicelulosa y FAD de los forrajes evaluados. Guanacaste, Costa Rica. 2000.

\section{Ganancia de peso}

En el Cuadro 4 se muestran los pesos finales de los animales. Dado que los pesos iniciales influencian los pesos finales de los animales, ambos se ajustaron por covarianza; de manera que para el análisis de varianza se utilizaron estos pesos finales ajustados.
Como se puede observar en el Cuadro 5 y la Figura 5, el peso final de los animales en el tratamiento con heno HBC, de pasto Transvala producido en sistemas bajo riego, fertilización y manejado a un rebrote de 45 días promedio, fue superior a los pesos de todos los animales alimentados con los otros forrajes.

Cuadro 4. Peso final en kilogramos de los animales. Guanacaste, Costa Rica. 2000.

\begin{tabular}{|c|c|c|c|c|c|c|c|c|c|}
\hline \multirow{3}{*}{$\begin{array}{c}\text { Rep. } \\
1\end{array}$} & \multicolumn{9}{|c|}{ Tratamiento } \\
\hline & \multicolumn{3}{|c|}{ HBC } & \multicolumn{3}{|c|}{$\mathrm{HCM}$} & \multicolumn{3}{|c|}{ PAR } \\
\hline & 346 & 324 & 343 & 362 & 342 & 295 & 314 & 297 & 284 \\
\hline 2 & 330 & 273 & 319 & 300 & 289 & 336 & 279 & 273 & 281 \\
\hline 3 & 279 & 290 & 307 & 218 & 274 & 212 & 248 & 293 & 291 \\
\hline Prom. & \multicolumn{3}{|c|}{312,3} & \multicolumn{3}{|c|}{292} & \multicolumn{3}{|c|}{284,4} \\
\hline PAC & \multicolumn{3}{|c|}{304,8} & \multicolumn{3}{|c|}{295,5} & \multicolumn{3}{|c|}{288,5} \\
\hline
\end{tabular}

PAC $=$ peso ajustado por covarianza. 
Cuadro 5. Comparación de Medias de Cuadrados Mínimos para peso final. Guanacaste, Costa Rica. 2000.

\begin{tabular}{|c|c|c|c|c|c|}
\hline \multirow{3}{*}{$\begin{array}{l}\text { Error estándar } \\
\text { de la media }\end{array}$} & \multirow{3}{*}{ Tratamiento } & \multirow{3}{*}{$\begin{array}{l}\text { Peso Final } \\
\text { (kg) }\end{array}$} & \multicolumn{3}{|c|}{ Tratamientos* } \\
\hline & & & & $\mathrm{HCM}$ & PAR \\
\hline & & & $304,8_{a}$ & $295,5_{b}$ & $288,5_{c}$ \\
\hline & Heno de Calidad & 304,8 & - & $(P<0,005)$ & $(\mathrm{P}<0,001)$ \\
\hline \multirow[t]{2}{*}{7,97} & Heno Comercial & 295,5 & & - & $(P<0,10)$ \\
\hline & Paja de Arroz & 288,5 & & & - \\
\hline
\end{tabular}

* Letras iguales indican que no hay diferencia significativa $(P \geq 0,05)$ entre tratamientos.

El mayor peso ganado por los animales del tratamiento HBC es aún más relevante si se consi-

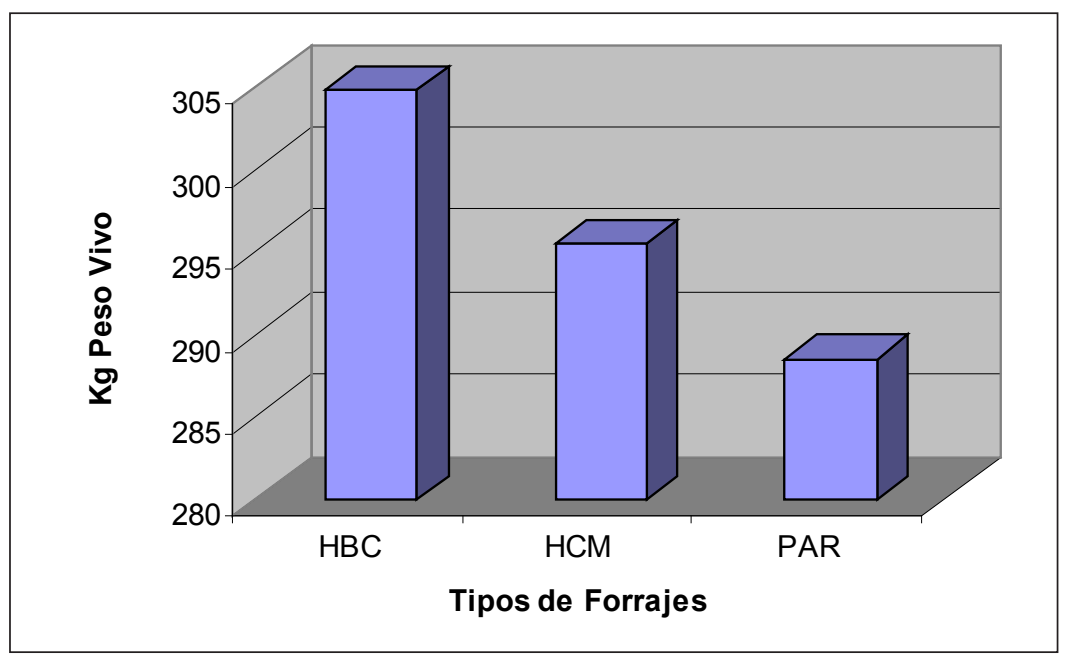

Figura 5. Peso final de animales alimentados con diferentes calidades de forrajes. Guanacaste, Costa Rica. 2000.

dera que no recibieron ningún otro ingrediente en la dieta, mientras que los animales de los otros dos tratamientos recibieron además del forraje, 0,5 kg de melaza (energía) y 30 gramos de urea como fuente de proteína cruda. Esto corresponde aproximadamente a 1,2 Mcal de energía digestible y 105 gramos de proteína cruda extra, respectivamente. El efecto positivo del suministro de nitrógeno no proteico como la urea y de fuentes energéticas como la melaza sobre la digestibilidad, el consumo y la producción, tanto en ganado de carne como de leche, alimentados con forrajes de baja calidad, está ampliamente demostrado en la literatura (Coleman y Barth 1977, Morales et al. 1989, Puoli et al.1991, Titgemeyer et al. 2004, Currier et al. 2004).

El propósito de suministrar estos nutrientes adicionales en el caso de los tratamientos del presente estudio, fue porque los animales alimentados con forrajes de mala calidad pierden peso, ya que no pueden consumir suficiente materia seca para satisfacer sus necesidades 
nutricionales de mantenimiento (Lippke 1980).

Además los ingredientes energéticos de fácil asimilación como la melaza y fuentes de nitrógeno no protéico como la urea, aportan nutrientes deficitarios en forrajes de baja calidad, permitiendo un mayor crecimiento de la población microbiana a nivel de rúmen, mejorando la utilización animal de dichos forrajes (Leng 1990, 1993), lo cual debe manifestarse en la producción animal.

Aún tomando en cuenta esta fuente de nutrientes extra, los resultados indican que las ganancias de peso de los animales alimentados con el heno de 45 días de rebrote fueron superiores a las de los animales alimentados con heno comercial y con paja de arroz.

\section{CONCLUSIONES Y RECOMENDACIONES}

1. La respuesta animal obtenida en el presente estudio confirma los resultados sobre el contenido nutricional de henos de diferentes calidades, encontrados en este y en estudios anteriores de esta serie (Morales et al. 2006 a y b).

2. El heno de buena calidad de pasto Transvala permite un mejor comportamiento productivo de los animales, debido al mayor contenido de nutrientes en este tipo de forrajes, lo cual se manifiesta en un mayor consumo y finalmente en mejores ganancias de peso. El heno de pasto Transvala puede ser de calidad superior al heno comercial, si se produce bajo condiciones de riego, se aplican fertilizantes, principalmente de tipo nitrogenado y si se controla la edad de rebrote a un promedio de 45 días.

3. Es importante destacar, tal como se indica en el objetivo del presente trabajo, que la estabulación de toretes con régimen alimenticio sólo con heno, como el realizado en el presente estudio, tuvo fines exclusivamente para probar y demostrar el impacto de la calidad del forraje sobre la producción animal. Por lo que en términos prácticos y comer- ciales, los animales estabulados deben recibir además de un forraje de buena calidad, otros ingredientes que les permitan obtener ganancias de peso muy superiores a las que se logran en sistemas bajo pastoreo.

4. Comercialmente, si se van a estabular animales, es porque se tiene el objetivo de producción sobre el kilo diario en ganancia de peso, con dietas balanceadas y de costos relativos aceptables, ya que la rentabilidad de este tipo de explotaciones es muy sensible a cambios de precio de los ingredientes y de venta de los animales.

\section{LITERATURA CITADA}

Arthington, J. D.; Brown, W. F. 2005. Estimation of feeding value of four tropical species al two stages of maturity. J. Anim. Sci. 83:1726-1731.

Coleman, S. W.; Barth, K. 1977. Utilization of supplemental NPN and energy sources by beef steers consuming low-protein hays. J. Anim. Sci. 45:1180-1187.

Currier, T. A.; Bohnert, D. W.; Schauer, S. J.; Bartle, S. J. 2004. Daily and alternate day supplementation of urea or biuret to ruminantes consuming lowquality forage: III. Effects on ruminal fermentation characteristics in stters. J. Anim. Sci. 82:1528-1535.

Cherney, J. H.; Hall, M. H. 2008. Forage quality in perspectiva. In: Forages. Agronomy Facts 30. Collage of Agricultural Sciences. Cooperative Extensión. PENNSTATE, $4 \mathrm{p}$.

Hancock, J. R.; Hargreaves, H. G. 1977. Precipitation, climate and potential for agricultural production Costa Rica. International Irrigation Center. Agricultural and Irrigation Engineering Dept. Utah State University. 50 p.

Jung, H. G.; Allen, M. S. 1995. Characteristics of plant cell walls affecting intake and 
digestibility of forages by ruminants. J. Anim. Sci. 73:2774 - 2790.

Leng, R. A. 1990. Factors affecting the utilization of poor quality forages by ruminant animals, particularly under tropical conditions. Nutrition Research Reviews. 3:277-303.

. 1993. Quantitative Ruminant Nutrition - A Green Science. Aust. J. Agri. Research 44:363-80.

Lippke, H.1980. Forage characteristics related to intake, digestibility and gain by ruminants. J. Anim. Sci., vol 50, №.5.

Morales, J. L.; Van Horn, H. H.; Moore, J. E. 1989. Dietary interaction of cane molasses with source of roughage: Intake and lactation effects. J. Dairy Sci. 72:2331-2338.

2000. Primer periodo seco del milenio. Revista Informativa del Comité de Educación y Bienestar Social. Año XV, No 104. p 6 y 7.

; Acuña, V.; Cruz, A. 2003. Industrialización del heno de calidad en sistemas bajo riego en Costa Rica. Imprenta Nacional. MAG. Costa Rica. p. 77.

; Cruz, A.; Acuña, V. 2006a. Efecto del estado de madurez y la fertilización nitrogenada sobre la producción y valor nutritivo del pasto transvala (Digitaria decumbens cv. Transvala) para henificación bajo condiciones de secano. Alcances Tecnológicos. Año IV, No 1. p. 37-44.

; Acuña, V.; Cruz, A. 2006b. Efecto de la fertilización nitrogenada sobre la producción y valor nutritivo del pasto Transvala (Digitaria decumbens cv. Transvala) para henificación, bajo condiciones de riego. Alcances Tecnológicos. Año IV, No 1. p. 45-51.

Puoli, J. R.; Jung, G. A.; Reid, R. L. 1991. Effects of nitrogen and sulphur on digestion and nutritive quality of warm-season grass hays for cattle and sheep. J. Anim. Sci. 69:843-852.

SAS INSTITUTE (Statistical Analisis System Institute, USA). 1984. SAS User's guide statistics. SAS Inst., Cary, NC.sp.

Titgemeyer, E. C.; Drouillard, J. S.; Greenwood, R. H.; Ringler, J. W.; Bindel, D. J.; Hunter R. D.; Nutsch, T. 2004. Effect of forage quality on digestion and performance responses of cattle to supplementation with cooked molasses blocks. J. Anim. Sci. 82:487-494.

Van Soest, P. J. 1967. Development of a comprehensive system of feed. Analyses and its application to forages. J. Anim. Sci. 26:119.

;1983. Nutritional Ecology of the Ruminant. O. \& B. Books. USA. sp.

\section{NOTA TÉCNICA}

